\title{
Communication
}

\section{Luminescent Sol-Gel Glasses from Silicate-Citrate-(Thio)Ureate Precursors}

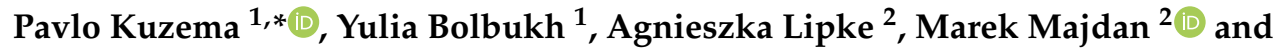 \\ Valentyn Tertykh ${ }^{1}$ \\ 1 Chuiko Institute of Surface Chemistry, National Academy of Sciences of Ukraine, 17 General Naumov Str., \\ 03164 Kyiv, Ukraine; yu_bolbukh@yahoo.com (Y.B.); tertykh@yahoo.com (V.T.) \\ 2 Faculty of Chemistry, Maria Curie-Skłodowska University, pl. Maria Curie-Skłodowskiej 2, 20-031 Lublin, \\ Poland; agalipke@gmail.com (A.L.); majdan.marek8@gmail.com (M.M.) \\ * Correspondence: coralchance@gmail.com; Tel.: +38-044-424-9474
}

Received: 9 December 2018; Accepted: 11 January 2019; Published: 14 January 2019

\begin{abstract}
Recent advancements in synthesis and analysis of the composites based on silica and carbon quantum dots have revealed great potential of such systems in bioimaging, sensor, as well as solid-state lighting applications. Most of the synthetic methods for obtaining such materials are still relatively complex and costly. The aim of this work was to study the luminescent properties of silica-based composites prepared by the simple sol-gel method using low-cost silicate-citrate-(thio)ureate precursors. The glassy composites were prepared by acid hydrolysis of ethyl silicate $(40 \%)$ in aqueous solution of citric acid ureates or thioureates with the citric acid-to-(thio)urea molar ratio of 1:1, 1:1.5 or 1:3. The results of spectrofluorimetric analysis have shown that heat-treated at $270{ }^{\circ} \mathrm{C}$ such silica gels upon UV excitation (with an optimum at $\lambda_{\text {exc }}=360 \mathrm{~nm}$ ) emit light in a visible spectrum $(400-600 \mathrm{~nm})$. Upon this, photoluminescence efficiency of ureate-derived glasses (quantum yield $70.53 \%$ for 1:1.5 sample) appeared to be much higher than that for thioureate-derived glasses (quantum yield $11.25 \%$ for $1: 3$ sample) suggesting that the preparation conditions to obtain the glasses with optimal photoluminescence characteristics are quite different in case of urea and thiourea. Thus, citrate-ureate-derived silica glasses already demonstrate very good potential to be efficient materials for different fluorescence-related applications.
\end{abstract}

Keywords: silica; citric acid; urea; thiourea; carbon dots; sol-gel; thermal treatment; luminescent properties

\section{Introduction}

Development of novel inexpensive environmentally friendly materials that convert UV light into visible light is of great importance, especially taking into account the fast growing solid-state lighting industry. In this respect, carbon-based quantum dots (CDs) are highly attractive due to their low toxicity, chemical inertness, high photostability and fluorescence [1]. Photoluminescence (PL) quantum yield (QY) for native CDs is usually quite low because of the presence of emission traps at the surface layer. It is necessary to form a passivation layer on the surface in order to improve their brightness. Also, higher PL QY can be achieved when layered and more crystalline graphene-based CDs are used [2]. Table 1 shows the PL properties of some CDs synthesized using various methods. 
Table 1. Photoluminescent characteristics of carbon dots synthesized by different methods.

\begin{tabular}{|c|c|c|c|}
\hline CD Size, $\mathrm{nm}$ & Synthetic Technique & PL QY, \% & Reference \\
\hline $2-4$ & Hydrothermal treatment of orange pericarp & up to 9 & [3] \\
\hline $5-9$ & $\begin{array}{l}\text { Solvothermal reaction between } \mathrm{SiCl}_{4} \text { and } \\
\text { hydroquinone (CDs, doped with } \mathrm{Si} \text { atoms) }\end{array}$ & 19 & [4] \\
\hline 5 & $\begin{array}{l}\text { CDs modification with oligo(ethylene glycol) diamine } \\
\text { (with subsequent doping with } \mathrm{ZnS}, \mathrm{ZnO} \text { or } \mathrm{TiO}_{2} \text { ) }\end{array}$ & 20 (up to 78 ) & [5] \\
\hline $2-4$ & $\begin{array}{l}\text { Heating of oligoethylenimine- } \beta \text {-cyclodextrin in } \\
\text { phosphoric acid }\end{array}$ & 30 & [6] \\
\hline$<10$ & $\begin{array}{l}\text { Thermal treatment of } O, O^{\prime} \text {-bis(3-aminopropyl) } \\
\text { polyethylene glycol (with further doping with } \mathrm{ZnS} \text { ) }\end{array}$ & $40(60)$ & [7] \\
\hline 5 & Hydrothermal method using urea and citric acid & 45 & [8] \\
\hline $3-4$ & $\begin{array}{l}\text { Hydrothermal oxidation of } \\
\text { branched polyethylenimine }\end{array}$ & 55 & [9] \\
\hline $2-5$ & $\begin{array}{l}\text { Heating of glucose with amino acids in } \\
\text { alkaline medium }\end{array}$ & up to 69 & [10] \\
\hline $5-9$ & $\begin{array}{l}\text { Hydrothermal synthesis using citric acid } \\
\text { and L-cysteine }\end{array}$ & 73 & [11] \\
\hline $2-3$ & $\begin{array}{l}\text { Hydrothermal synthesis using citric acid and urea } \\
\text { (or thiourea) }\end{array}$ & $78(71)$ & [12] \\
\hline
\end{tabular}

Most of these methods are not quite satisfactory because of the necessity to use costly equipment, the complexity of synthesis procedure, or low PL QY. In particular, in the majority of cases, the CDs obtained demonstrate relatively low PL QY (usually, less than 50\%). In order to improve the CDs brightness, their doping with some salts and oxides has been proposed [5,7]. For instance, ZnS-doped CDs with passivation using the molecules of oligomeric poly(ethylene glycol) diamine (PEG1500N), after column gel fractionation demonstrate PL QY 78\% [5]. However, the procedure of such CDs synthesis is relatively complex. Among the variety of methods to obtain highly luminescent CDs it is worth noting the hydrothermal synthesis of $\mathrm{N}$ - or N,S-doped CDs, using citric acid (C-source) and urea (N-source) or thiourea/L-cysteine (N,S-sources) [11,12]. The main advantages of this synthetic route are the low cost and the ease of implementation and scaling, whereas PL QY of the CDs obtained reaches $70-78 \%$.

Taking into account that the conditions for production of inexpensive environmentally friendly high-luminescent materials are still in development, as well as because of the need to enlarge the application area of CDs and to restrain their solid-state PL quenching, further development of the synthetic routes to obtain novel CD-based composites with improved PL characteristics is relevant. In this aspect, a promising approach is the formation of CDs with doped heteroatoms either on the surface or inside the bulk of oxide materials, in particular silica. On the one hand, silica as a substrate, will localize CDs growth, blocking the agglomeration of nanoparticles during heat treatment, on the other hand, the doping of CDs with silicon contributes to the increase of the PL characteristics of the CDs. Besides, it has been shown that silica gel glasses produced from silicate carboxylate precursors exhibit high PL characteristics [13]. Recent advancements in synthesis and analysis of silica/CDs composites revealed great potential of such systems in bioimaging, sensor, as well as solid-state lighting applications, and depending on the synthesis methods and the precursors used, it is possible already to achieve the PL QY from typically 7-14 up to 60\% [14-22]. Table 2 gives the PL characteristics of some carbon-containing silica-based materials synthesized using various methods. 
Table 2. Photoluminescent characteristics of carbon-containing silica-based materials synthesized by different methods.

\begin{tabular}{|c|c|c|c|}
\hline CD Size, nm & Synthetic Technique & PL QY, \% & Reference \\
\hline- & $\begin{array}{l}\text { Thermal treatment of sol-gel glasses from } \\
\text { tetraalkoxysilane or 3-aminopropyltriethoxysilane } \\
\text { (APTES) and various carboxylic acids }\end{array}$ & up to 45 & [13] \\
\hline $1.3-1.7$ & $\begin{array}{l}\text { Heat treatment of sol-gel materials from } \\
\text { tetraethoxysilane (TEOS) and APTES in the presence of } \\
\text { hexadecyltrimethylammonium bromide }\end{array}$ & up to 60 & [14] \\
\hline 8 & $\begin{array}{l}\text { Sol-gel synthesis from organoalkoxysilanes in the } \\
\text { presence of CDs obtained via the hydrothermal } \\
\text { synthesis using citric acid and ethylenediamine }\end{array}$ & $9-14$ & [17] \\
\hline 4.5 & $\begin{array}{l}\text { TEOS hydrolysis in the presence of glucose and } \\
\text { 1,2-ethylenediamine }\end{array}$ & up to 13 & [19] \\
\hline- & $\begin{array}{c}\text { Sol-gel synthesis from TEOS in the presence of CDs } \\
\text { obtained via the hydrothermal synthesis using citric acid } \\
\text { and ethylenediamine }\end{array}$ & 21.5 & [20] \\
\hline $4-9$ & $\begin{array}{l}N \text {-(3-(trimethoxysilyl) propyl) ethylenediamine } \\
\text { hydrolysis in the presence of CDs obtained by } \\
\text { solvothermal treatment of } p \text {-phenylenediamine }\end{array}$ & 41.72 & [21] \\
\hline- & $\begin{array}{l}\text { Pyrolysis of citric acid (thio)ureates at the fumed } \\
\text { silica surface }\end{array}$ & $7-11$ & [22] \\
\hline
\end{tabular}

Most of the synthetic methods are still relatively complex and costly or the materials produced possess relatively low PL QY. In [22], the simple and inexpensive route to produce luminescent silica-based nanomaterials was used: citric acid ureates were deposited from aqueous or ethanol solutions onto fumed silica surface. After drying and further thermal treatment at the temperature of up to $270{ }^{\circ} \mathrm{C}$, such materials were found to demonstrate fluorescent properties, however, PL QY was quite low (within $7-11 \%$ ). The aim of this work was to further develop this synthetic approach by preparing sol-gel silica-based composites from silicate citrate (thio)ureate precursors and by investigating their PL characteristics.

\section{Materials and Methods}

Silica-based composites were prepared by acid hydrolysis of ethyl silicate $(40 \%)$ in aqueous solution of citric acid ureates or thioureates with the citric acid-to-(thio)urea molar ratio of 1:1, 1:1.5 or 1:3. The typical procedure of synthesis was as follows: A total of $83 \mathrm{mg}$ of citric acid and $26 \mathrm{mg}$ of urea or $33 \mathrm{mg}$ of thiourea (all the reagents are of chemical purity grade) were added into a glass with $5 \mathrm{~mL}$ of distilled water, and then the mixture was stirred with a magnetic stirrer until the complete dissolution of the reagents. After that, the obtained solution of citric acid mono(thio)ureate was added to a solution of ethyl silicate acidified with concentrated hydrochloric acid solution. Full quantitative description of the components used for all the samples preparation is given in Table 3.

The prepared sols were left to stir overnight. Then the samples were dried at $60{ }^{\circ} \mathrm{C}$ for $3 \mathrm{~h}$ and at $105^{\circ} \mathrm{C}$ for another $3 \mathrm{~h}$. Obtained transparent glassy gels were further thermally treated with gradual temperature increase up to $270{ }^{\circ} \mathrm{C}$ (the heating rate was about $1{ }^{\circ} \mathrm{C} / \mathrm{min}$ ) and maintained at $270{ }^{\circ} \mathrm{C}$ for additional $1 \mathrm{~h}$. After cooling to room temperature, the resulted amber-to-dark brown colored glassy monoliths were powdered in the agate mortar and subjected to spectrofluorimetric analysis. Fluorescence spectra were obtained using a Photon Technology International spectrofluorimeter equipped with a continuous wave xenon arc (Xe-arc) lamp as a light source. Spectral resolution was maintained at $1 \mathrm{~nm}$. The absolute quantum yields of the fluorescence of solid samples were determined using a K-Sphere "Petite" integrating sphere (Photon Technology International, Inc., Edison, NJ, USA). 
Table 3. Sample preparation: components ratio.

\begin{tabular}{cccccccc}
\hline Sample & $\begin{array}{c}\text { Ur(ThUr):CA } \\
\text { Molar Ratio }\end{array}$ & $\begin{array}{c}\text { V(ES-40), } \\
\mathbf{m L}\end{array}$ & $\begin{array}{c}\mathbf{V}\left(\mathbf{H}_{\mathbf{2}} \mathbf{O}\right), \\
\mathbf{m L}\end{array}$ & $\begin{array}{c}\mathbf{m}(\mathrm{CA}), \\
\mathbf{m g}\end{array}$ & $\begin{array}{c}\mathbf{m}(\mathrm{Ur}), \\
\mathbf{m g}\end{array}$ & $\begin{array}{c}\mathbf{m} \text { (ThUr), } \\
\mathbf{m g}\end{array}$ & $\begin{array}{c}\mathrm{V}(\mathbf{H C l}), \\
\mathbf{m L}\end{array}$ \\
\hline 1 & $1: 1$ & 25 & 5 & 83 & 26 & & 0.3 \\
2 & $1.5: 1$ & 25 & 5 & 82 & 38 & & 0.3 \\
3 & $3: 1$ & 25 & 5 & 64.5 & 60.5 & & 0.3 \\
4 & $1: 1$ & 25 & 5 & 83 & & 33.3 & 0.3 \\
5 & $1.5: 1$ & 25 & 5 & 83 & & 50 & 0.3 \\
6 & $3: 1$ & 25 & 5 & 64.5 & & 77.7 & 0.3 \\
\hline
\end{tabular}

$\mathrm{Ur}=$ urea, $\mathrm{ThUr}=$ thiourea, $\mathrm{CA}=$ citric acid, ES-40 = ethyl silicate $(40 \%)$.

\section{Results and Discussion}

Formation of silica glasses by the acid-catalyzed sol-gel route proceeds through several basic steps: (1) partial hydrolysis of liquid ethyl silicate precursor; (2) polycondensation of formed colloid-like oligomers; (3) additional hydrolysis which promotes polymerization and crosslinking leading to a three-dimensional matrix formation. All the reactions may occur simultaneously (Scheme 1).

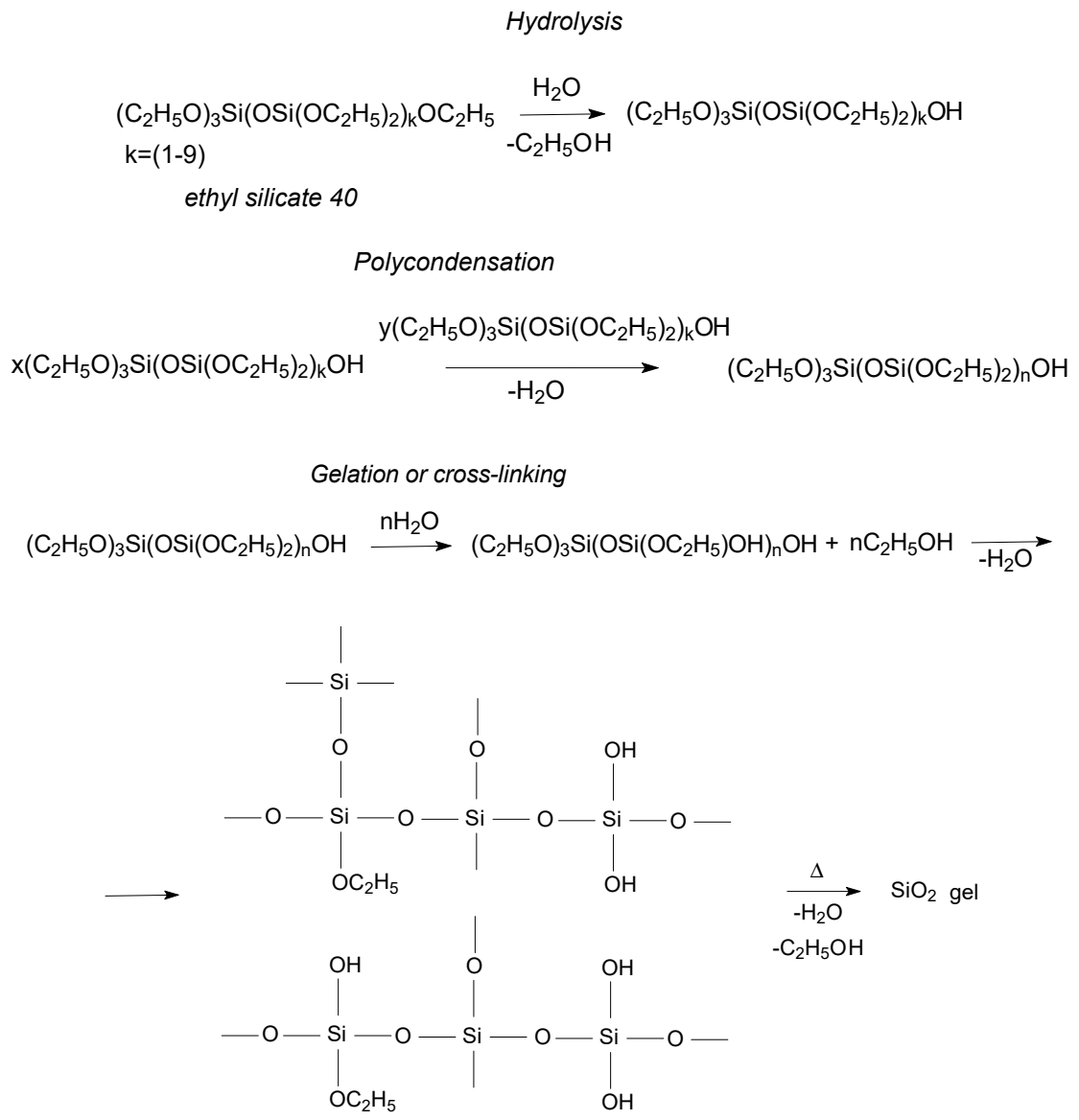

Scheme 1. The process of gel formation by ethyl silicate hydrolysis.

After drying at $60^{\circ} \mathrm{C}$ and then at $105^{\circ} \mathrm{C}$, the resulting transparent silica-based glasses (presumably containing citric acid (thio)ureates condensed inside the silica matrix) were crushed and preliminary tested on luminescent properties with UV-blue flashlight. Blue and in some cases greenish glow were observed suggesting no notable white luminescence from the samples (Figures S1 and S2, Supplementary Materials). In contrast to that, after thermal treatment at $270{ }^{\circ} \mathrm{C}$ the resulted amber-to-dark brown glasses under UV-blue flashlight irradiation demonstrate notable fluorescence (Figures S1 and S2), and even a phosphorescence in the case of ureates. At this stage of preliminary 
analysis the citrate ureate-derived silica-based glasses seemed to possess brighter luminescence than those from citrate thioureate precursors.

Figure 1 gives excitation and emission PL spectra for the samples of thermally treated at $270{ }^{\circ} \mathrm{C}$ silica gel which contained the salts with citric acid-to-urea ratio 1:1, 1:1.5 and 1:3. As one can see, the range of wavelengths $325-375 \mathrm{~nm}$ is the most efficient for excitation of luminescent emission in the visible region (for the case of 450 and $500 \mathrm{~nm}$ wavelengths detection), the maximum being at about $360 \mathrm{~nm}$ (Figure 1a,b). Under UV irradiation with excitation wavelengths $\lambda_{\text {exc }}=335 \mathrm{~nm}$ and $360 \mathrm{~nm}$, the maximum luminescence is in the area of 430-520 nm (two PL maxima at about 445 $\mathrm{nm}$ and $500 \mathrm{~nm}$, Figure 1c,d), that is, a phosphor red shifts the radiation spectrum, demonstrating nearly excitation-independent PL. Such type of PL (within $\lambda_{\mathrm{exc}}=340-400 \mathrm{~nm}$ ) is also a characteristic for the aqueous solution of graphene-based $\mathrm{N}$-doped CDs prepared via the hydrothermal synthesis route using citric acid and urea as the precursors [12]. The optimal $\lambda_{\text {exc }}$ for those objects was also $360 \mathrm{~nm}$, however, the difference is that only one PL maximum was observed-at $435 \mathrm{~nm}$. The authors implied [12] that excitation-independent PL should originate from the uniformity of both the size and the surface state of $\mathrm{sp}^{2}$ clusters contained in graphene-based quantum dots. The proposed mechanism of graphene $C D$ s formation envisages citric acid self-assembling into nanosheets structure (due to intermolecular $\mathrm{H}$-bonding) and then dehydrolysis to give graphene nanoparticles with lots of carboxyl and carbonyl groups at the surface (O-states). The surface states (O-states and $\mathrm{N}$-states in the case of N-doped CDs) are supposed to be responsible for the PL mechanism [11]: during UV irradiation electrons are excited from the ground state and trapped by the surface states, high density of doped $\mathrm{N}$-states being able to facilitate a high yield of radiative recombination when excited electrons return to the ground state. When the density of $\mathrm{N}$-states is higher than that of the O-states (or N-states are enhanced and/or O-states are suppressed like in the case of N,S-doped CDs), bright excitation-independent PL occurs. Slight red shift of first PL maximum ( $445 \mathrm{~nm}$ vs. $435 \mathrm{~nm}$ ) in our case may be related to the influence of $\mathrm{SiO}_{2}$ environment suggesting that some $\mathrm{Si}-\mathrm{O}-\mathrm{C}$ bonding with incorporated carbon-based structures may occur. This influence of silica matrix becomes even more evident if one analyzes the second PL maximum at $500 \mathrm{~nm}$ (Figure 1c,d). Excitation-independent PL with maximum at about $550 \mathrm{~nm}$ was also observed for CDs obtained by condensation between tetraethoxysilane and glucose in the presence of 1,2-ethylenediamine [19]. It was assigned to the confined growth of CDs in silica network. The Si-O-C linkage at the CDs surface is known to be required to induce PL of C-doped silica materials, and, therefore, "glucose was a required component to produce the chromophore and silica backbone, such as auxochrome, significantly affecting both intensity and wavelength of the fluorescence" [19]. In our case, under anhydrous conditions (when water was eliminated from the gel), citric acid may interact (via the carboxyl groups, Scheme 2) with residual ethoxysilyl groups and in substantial amount may be retained in the gel as a silyl citrate species:

Upon heating, this citrate may either participate in CDs growth process (by the mechanism described above) to produce luminescent carbon-based structures with Si-O-C links, or it may decompose to create the $\mathrm{C}$ substitutional defects for $\mathrm{Si}$, which are also assumed to be the luminescent species in the lattice. The scheme of PL mechanism for the simplest case of Si substitution with C is shown below (Scheme 3). 


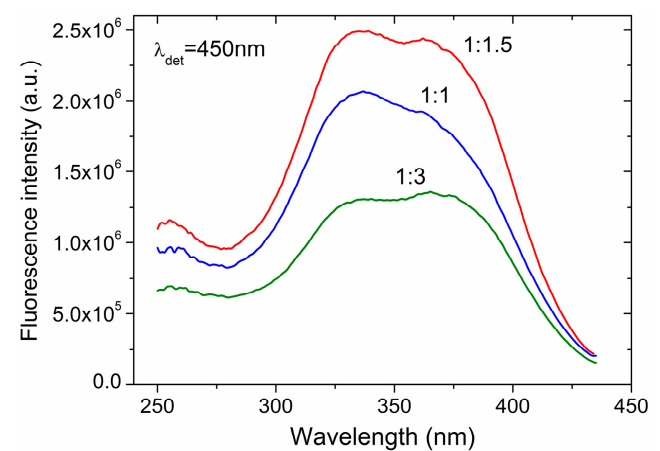

(a)

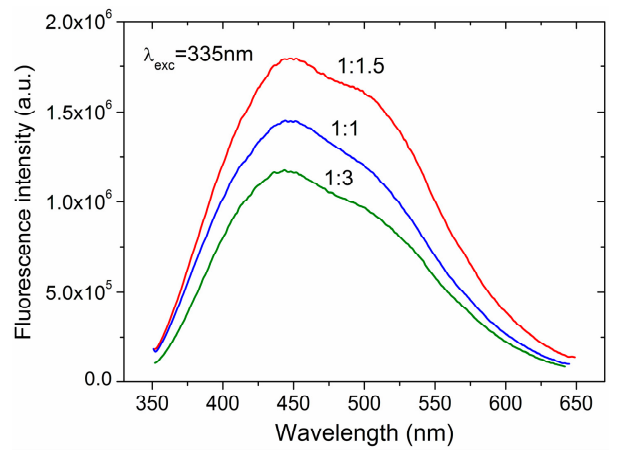

(c)

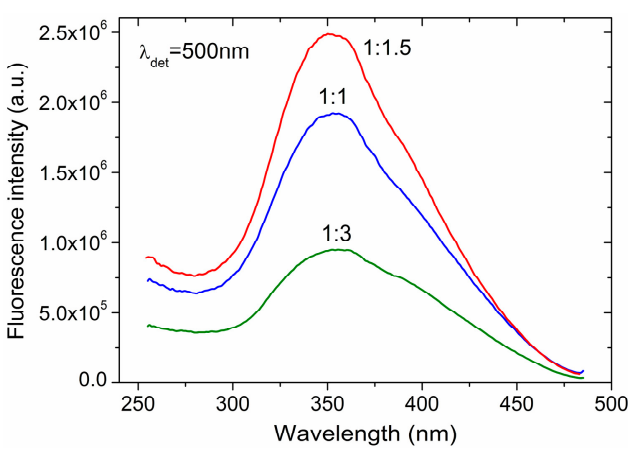

(b)

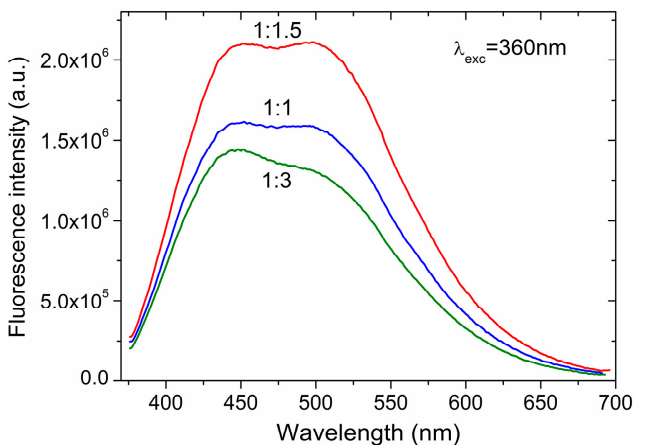

(d)

Figure 1. Excitation $(\mathbf{a}, \mathbf{b})$ and emission $(\mathbf{c}, \mathbf{d})$ spectra of the composites based on silica and citric acid ureates $\left(1: 1,1: 1.5\right.$ and 1:3) after thermal treatment at $270{ }^{\circ} \mathrm{C}$. ( $\lambda_{\text {det }}$-detection wavelength, $\lambda_{\text {exc }}$-excitation wavelength).

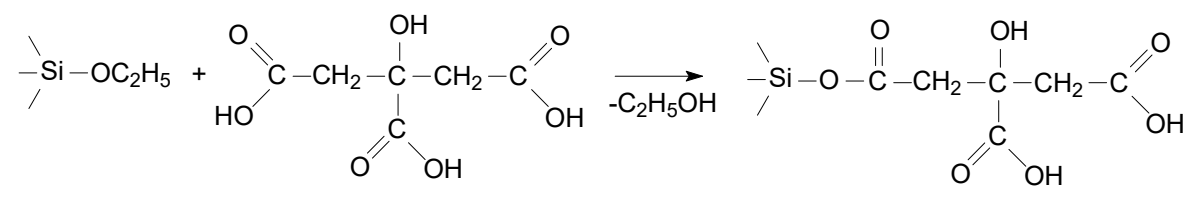

Scheme 2. Interaction of citric acid with ethoxysilyl groups.

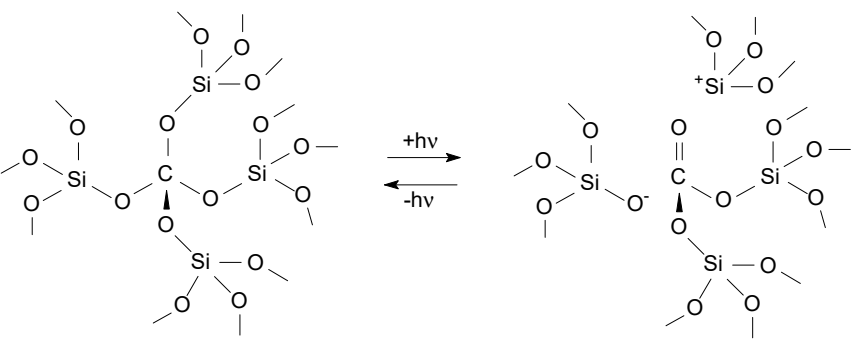

Scheme 3. PL mechanism in the case of $\mathrm{C}$ substitutional defects for $\mathrm{Si}$ in the $\mathrm{SiO}_{2}$ lattice.

In order to confirm the assumption that citric acid interaction with ethoxysilyl groups could contribute to the second PL peak, we compared the PL spectra for the silica-based glasses prepared from the precursors with different citric acid-to-urea ratios (Figure 1). At 1:1.5 ratio no significant qualitative changes were found, but at 1:3 ratio, when there is enough amino groups of urea molecules to engage all the carboxyl groups of citric acid molecules into 1:1 interaction, the first PL peak became more intense than the second one (Figure 1d). Thus, we attribute the first PL peak to the carbon-based luminescent species which are not or weakly bound to silica backbone and, like $\mathrm{N}$-doped graphene-based CDs obtained via the hydrothermal synthesis, demonstrate bright blue excitation-independent luminescence when exposed to UV irradiation. The second PL peak is 
apparently due to the luminescent carbon-based species bound to silica matrix via Si-O-C links, which demonstrate green-yellow excitation-independent luminescence when exposed to UV irradiation. Upon this, one may alter the luminescent properties by changing the citric acid-to-urea ratio. The PL QY for the sample with the highest PL intensity observed (1:1.5, Figure 1) was determined to be $70.53 \%$. This value is close to the one determined for aqueous solution of $\mathrm{N}$-doped CDs produced via the hydrothermal route (78\% [12]). This result suggests that the conditions for obtaining luminescent silica-based glasses from silicate citrate ureate precursors were close to optimal. Moreover, unlike pure $\mathrm{N}$-doped CDs, these materials possess much higher possibilities for fluorescence tuning and, therefore, they are much more promising for different fluorescence-related applications, especially solid-state lighting.

Substitution of ureate with thioureate at citric acid-to-thiourea ratio of 1:1 leads to qualitative changes in the luminescence spectrum (Figure 2). UV excitation with $\lambda=360 \mathrm{~nm}$ remains the optimal for PL in the visible region, but the maximum luminescence is in the region of 500-550 nm (one PL peak with $\lambda_{\max }$ at about $507-520 \mathrm{~nm}$ with a shoulder at $445 \mathrm{~nm}$, Figure $1 \mathrm{c}, \mathrm{d}$ ), that is, a phosphor red shifts the radiation spectrum, demonstrating nearly excitation-independent PL in the blue region, but in the green region, where emission peak is located, PL is excitation-dependent $\left(\lambda_{\max }=507 \mathrm{~nm}\right.$ at $\lambda_{\text {exc }}=335 \mathrm{~nm}$ and $\lambda_{\max }=517 \mathrm{~nm}$ at $\lambda_{\text {exc }}=360 \mathrm{~nm}$ ). Weakly expressed PL maximum in the blue region, like in the case of urea, can be attributed to the carbon-based luminescent species which are not or weakly bound to silica matrix and are formed in much less amount than in the case of urea. The PL maximum in the green region is probably due to the luminescent carbon-based species bound to silica matrix via Si-O-C links. These species demonstrate green-yellow excitation-dependent luminescence when exposed to UV irradiation, suggesting that their size and surface states are not uniform. Such a difference in luminescent characteristics of thioureate- in comparison with ureate-derived silica glasses may be explained if one assumes weaker thiourea (than urea) interaction with citric acid and, therefore, stronger interaction of citric acid with residual ethoxysilyl groups during the glasses formation and thermal treatment. This assumption is supported when we compare the PL spectra for the silica-based glasses prepared from the precursors with different citric acid-to-thiourea ratios (Figure 2). Indeed, notable qualitative changes were not observed in the spectra even at 3 times higher thiourea concentration when there is enough amino groups of thiourea molecules to engage all the carboxyl groups of citric acid molecules into 1:1 interaction (Figure 2c,d). Among the samples where thiourea was used, the PL QY for the sample with the highest PL intensity observed (1:3, Figure 2) was determined to be $11.25 \%$. This value is much less than that determined for aqueous solution of N,S-doped CDs produced via the hydrothermal route (71\% [12]). This result suggests that the conditions for obtaining highly luminescent silica-based glasses from silicate citrate thioureate precursors were far from optimal. In particular, the temperature of glasses pyrolysis was probably too high. Literature analysis suggests that starting from $140{ }^{\circ} \mathrm{C}$ thiourea isomerizes into thiocyanate, and above $180{ }^{\circ} \mathrm{C}$ significant $\mathrm{S}$ loss occurs along with the release of a large amount of gaseous products [23]. At $270{ }^{\circ} \mathrm{C}$ the main solid products of thiourea decomposition are $\mathrm{N}$-containing heterocyclic compounds melam and melem [23]. The latter was found to possess the bright luminescent properties (PL QY being up to 56.9\%) [24], however, if it is present in silica matrix, its efficiency is not so high as in the bulk state.

Thus, heat-treated at $270{ }^{\circ} \mathrm{C}$ silica glasses, prepared by acid hydrolysis of ethyl silicate from aqueous-alcohol solutions in the presence of citric acid ureates or thioureates (with citric acid-to-(thio)urea ratio 1:1, 1:1.5 or 1:3), upon UV excitation (with an optimum at $\lambda_{\text {exc }}=360 \mathrm{~nm}$ ) emit light in a visible spectrum $(400-600 \mathrm{~nm}$ ). Upon this, PL efficiency of ureate-derived glasses (PL QY $70.53 \%$ for 1:1.5 sample) appeared to be much higher than that for thioureate-derived glasses (PL QY $11.25 \%$ for $1: 3$ sample) suggesting that the preparation conditions to obtain the materials with optimal PL characteristics are quite different in case of urea and thiourea. Citrate-ureate-derived silica glasses already demonstrate very good potential to be efficient materials for different fluorescence-related applications, however, to obtain sol-gel glasses from silicate-citrate-(thio)ureate precursors with 
optimized and tunable PL parameters, as well as to get more insight into the PL mechanism and factors affecting these PL parameters, further, more systematic research needs to be carried out.

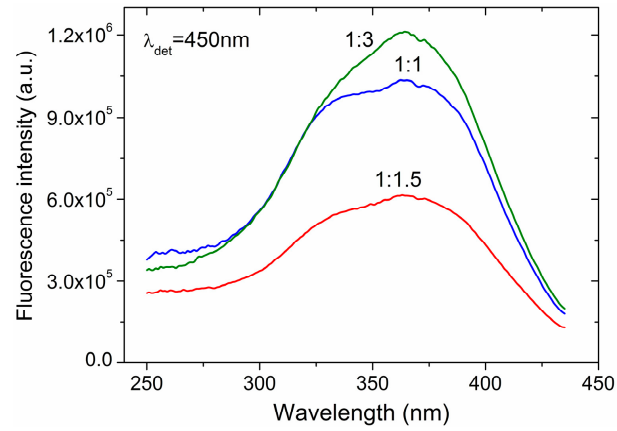

(a)

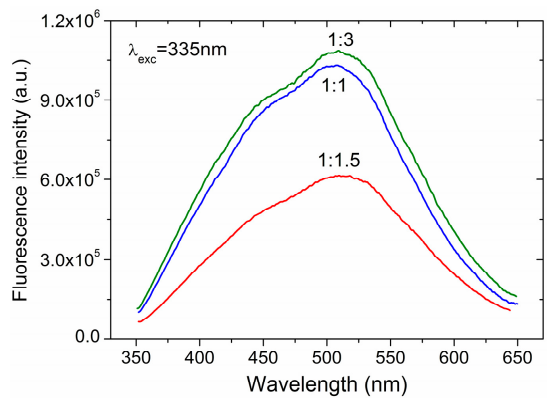

(c)

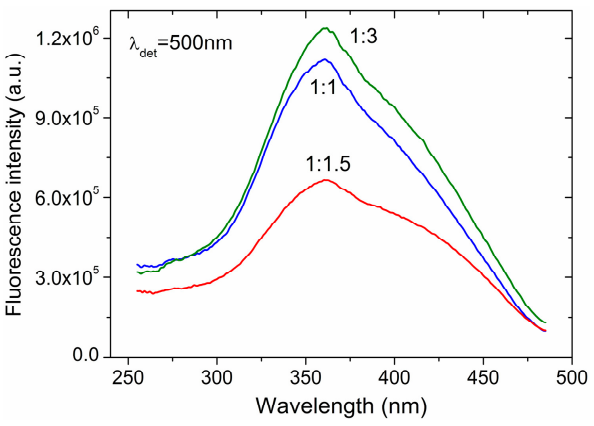

(b)

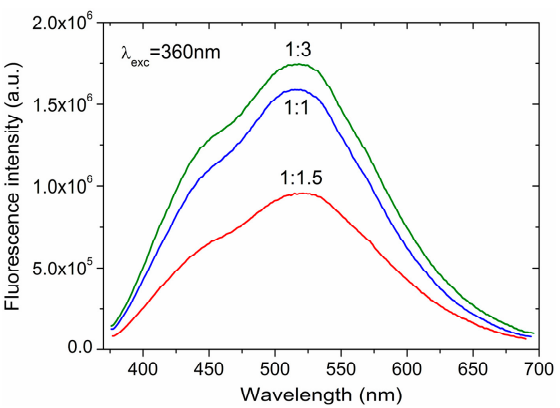

(d)

Figure 2. Excitation $(\mathbf{a}, \mathbf{b})$ and emission $(\mathbf{c}, \mathbf{d})$ spectra of the composites based on silica and citric acid thioureates $(1: 1,1: 1.5$ and $1: 3)$ after thermal treatment at $270{ }^{\circ} \mathrm{C}$.

Supplementary Materials: The following are available online at http:/ / www.mdpi.com/2504-5377/3/1/11/s1, Figure S1: photographs of the sol-gel glasses obtained from silicate-citrate-ureate precursors. From left to right: citric acid-to-urea ratio 1:1, 1:1.5, 1:3. Top down: dried at $105^{\circ} \mathrm{C}$ and exposed to white flashlight irradiation; dried at $105^{\circ} \mathrm{C}$ and exposed to UV-blue flashlight irradiation; thermally treated at $270{ }^{\circ} \mathrm{C}$ and exposed to white flashlight irradiation; thermally treated at $270^{\circ} \mathrm{C}$ and exposed to UV-blue flashlight irradiation, Figure S2: photographs of the sol-gel glasses obtained from silicate-citrate-thioureate precursors. From left to right: citric acid-to-thiourea ratio 1:1, 1:1.5, 1:3. Top down: dried at $105^{\circ} \mathrm{C}$ and exposed to white flashlight irradiation; dried at $105^{\circ} \mathrm{C}$ and exposed to UV-blue flashlight irradiation; thermally treated at $270{ }^{\circ} \mathrm{C}$ and exposed to white flashlight irradiation; thermally treated at $270{ }^{\circ} \mathrm{C}$ and exposed to UV-blue flashlight irradiation.

Author Contributions: Conceptualization, P.K. and Y.B.; Investigation, P.K., Y.B. and A.L.; Methodology, P.K. and A.L.; Supervision, M.M. and V.T.; Writing-original draft, P.K.; Writing-review \& editing, M.M. and V.T.

Funding: This research received no external funding.

Conflicts of Interest: The authors declare no conflict of interest.

\section{References}

1. Yang, Z.; Li, Z.; Xu, M.; Ma, Y.; Zhang, J.; Su, Y.; Gao, F.; Wei, H.; Zhang, L. Controllable synthesis of fluorescent carbon dots and their detection application as nanoprobes. Nano-Micro. Lett. 2013, 5, 247-259. [CrossRef]

2. Zheng, X.T.; Ananthanarayanan, A.; Luo, K.Q.; Chen, P. Glowing graphene quantum dots and carbon dots: Properties, syntheses, and biological applications. Small 2015, 11, 1620-1636. [CrossRef] [PubMed]

3. Du, W.; Xu, X.; Hao, H.; Liu, R.; Zhang, D.; Gao, F.; Lu, Q. Green synthesis of fluorescent carbon quantum dots and carbon spheres from pericarp. Sci. China Chem. 2015, 58, 863-870. [CrossRef]

4. Qian, Z.; Shan, X.; Chai, L.; Ma, J.; Chen, J.; Feng, H. Si-doped carbon quantum dots: A facile and general preparation strategy, bioimaging application, and multifunctional sensor. ACS Appl. Mater. Interfaces 2014, 6, 6797-6805. [CrossRef] [PubMed] 
5. Anilkumar, P.; Wang, X.; Cao, L.; Sahu, S.; Liu, J.-H.; Wang, P.; Korch, K.; Tackett, K.N.; Parenzan, A.; Sun, Y.-P. Toward quantitatively fluorescent carbon-based "quantum" dots. Nanoscale 2011, 3, 2023-2027. [CrossRef]

6. Yang, C.; Thomsen, R.P.; Ogaki, R.; Kjems, J.; Teo, B.M. Ultrastable green fluorescence carbon dots with high quantum yield for bioimaging and use as theranostic carriers. J. Mater. Chem. B 2015, 3, 4577-4584. [CrossRef]

7. Cao, L.; Yang, S.-T.; Wang, X.; Luo, P.G.; Liu, J.-H.; Sahu, S.; Liu, Y.; Sun, Y.-P. Competitive performance of carbon "quantum" dots in optical bioimaging. Theranostics 2012, 2, 295-301. [CrossRef]

8. Li, X.; Zhang, S.; Kulinich, S.A.; Liu, Y.; Zeng, H. Engineering surface states of carbon dots to achieve controllable luminescence for solid-luminescent composites and sensitive $\mathrm{Be}^{2+}$ detection. Sci. Rep. 2014, 4, 4976. [CrossRef]

9. Hu, L.; Sun, Y.; Li, S.; Wang, X.; Hu, K.; Wang, L.; Liang, X.; Wu, Y. Multifunctional carbon dots with high quantum yield for imaging and gene delivery. Carbon 2014, 67, 508-513. [CrossRef]

10. Wei, W.; Xu, C.; Wu, L.; Wang, J.; Ren, J.; Qu, X. Non-enzymatic-browning-reaction: A versatile route for production of nitrogen-doped carbon dots with tunable multicolor luminescent display. Sci. Rep. 2014, 4, 3564. [CrossRef]

11. Dong, Y.; Pang, H.; Yang, H.B.; Guo, C.; Shao, J.; Chi, Y.; Li, C.M.; Yu, T. Carbon-based dots co-doped with nitrogen and sulfur for high quantum yield and excitation-independent emission. Angew. Chem. Int. Ed. 2013, 52, 1-6. [CrossRef] [PubMed]

12. Qu, D.; Zheng, M.; Du, P.; Zhou, Y.; Zhang, L.; Li, D.; Tan, H.; Zhao, Z.; Xied, Z.; Sun, Z. Highly luminescent $\mathrm{S}, \mathrm{N}$ co-doped graphene quantum dots with broad visible absorption bands for visible light photocatalysts. Nanoscale 2013, 5, 12272-12277. [CrossRef] [PubMed]

13. Green, W.H.; Le, K.P.; Grey, J.; Au, T.T.; Sailor, M.J. White phosphors from a silicate-carboxylate sol-gel precursor that lack metal activator ions. Science 1997, 276, 1826-1828. [CrossRef]

14. Singh, R.K.; Patel, K.D.; Mahapatra, C.; Kang, M.S.; Kim, H.-W. C-Dot generated bioactive organosilica nanospheres in theranostics: multicolor luminescent and photothermal properties combined with drug delivery capacity. ACS Appl. Mater. Interfaces 2016, 8, 24433-24444. [CrossRef] [PubMed]

15. Wang, Z.; Xu, C.; Lu, Y.; Wu, F.; Ye, G.; Wei, G.; Sun, T.; Chen, J. Visualization of adsorption: Luminescent mesoporous silica-carbon dots composite for rapid and selective removal of $\mathrm{U}(\mathrm{VI})$ and in situ monitoring the adsorption behavior. ACS Appl. Mater. Interfaces 2017, 9, 7392-7398. [CrossRef]

16. Xiang, G.; Ren, Y.; Zhang, H.; Fan, H.; Jiang, X.; He, L.; Zhao, W. Carbon dots based dual-emission silica nanoparticles as ratiometric fluorescent probe for chromium speciation analysis in water samples. Can. J. Chem. 2018, 96, 72-77. [CrossRef]

17. Suzuki, K.; Malfatti, L.; Takahashi, M.; Carboni, D.; Messina, F.; Tokudome, Y.; Takemoto, M.; Innocenzi, P. Design of carbon dots photoluminescence through organo-functional silane grafting for solid-state emitting devices. Sci. Rep. 2017, 7, 5469. [CrossRef]

18. Nelson, D.K.; Razbirin, B.S.; Starukhin, A.N.; Eurov, D.A.; Kurdyukov, D.A.; Stovpiaga, E.Yu.; Golubev, V.G. Photoluminescence of carbon dots from mesoporous silica. Opt. Mater. 2016, 59, 28-33. [CrossRef]

19. Tian, Y.; Ran, Z.; Yang, W. Carbon dot-silica composite nanoparticle: An excitation-independent fluorescence material with tunable fluorescence. RSC Adv. 2017, 7, 43839-43844. [CrossRef]

20. Guo, Z.; Zhu, Zh.; Zhang, X.; Chen, Y. Facile synthesis of blue-emitting carbon dots@mesoporous silica composite spheres. Solid State Sci. 2018, 76, 100-104. [CrossRef]

21. Wang, J.; Zhang, F.; Wang, Y.; Yang, Y.; Liu, X. Efficient resistance against solid-state quenching of carbon dots towards white light emitting diodes by physical embedding into silica. Carbon 2018, 126, 426-436. [CrossRef]

22. Kuzema, P.O.; Bolbukh, Yu.M.; Tertykh, V.A. Luminescent materials based on organic salts pyrolyzed at the silica surface. Him. Fiz. Tehnol. Poverhni 2018, 9, 404-410. [CrossRef]

23. Timchenko, V.; Novozhilov, A.; Slepysheva, O. Kinetics of thermal decomposition of thiourea. Russ. J. Gen. Chem. 2004, 74, 1046-1050. [CrossRef]

24. Zheng, H.; Chen, W.; Gao, H.; Wang, Y.; Guo, H.; Guo, S.; Tang, Z.; Zhang, J. Melem: An efficient metal-free luminescence material. J. Mater. Chem. C 2017, 5, 10746-10753. [CrossRef]

(C) 2019 by the authors. Licensee MDPI, Basel, Switzerland. This article is an open access article distributed under the terms and conditions of the Creative Commons Attribution (CC BY) license (http:/ / creativecommons.org/licenses/by/4.0/). 